\title{
Role of the lysyl oxidase family in organ development (Review)
}

\author{
SHANZUN WEI ${ }^{1,2^{*}}$, LIANG GAO $^{1,2^{*}}$, CHANGJING WU $^{1}$, FENG QIN ${ }^{1}$ and JIUHONG YUAN ${ }^{1,2}$ \\ ${ }^{1}$ Andrology Laboratory and ${ }^{2}$ Department of Urology, West China Hospital, \\ Sichuan University, Chengdu, Sichuan 610041, P.R. China
}

Received September 18, 2019; Accepted March 2, 2020

DOI: $10.3892 /$ etm.2020.8731

\begin{abstract}
Lysyl oxidase proteins (LOXs) are amine oxidases, which are mainly located in smooth muscle cells and fibroblasts and serve an important role in the formation of the extracellular matrix (ECM) in a copper-dependent manner. Owing to the ability of LOX proteins to modulate crosslinking between collagens and to promote the deposition of other fibers, they serve crucially in organogenesis and the subsequent organ development, as well as disease initiation and progression. In addition, ECM formation significantly influences organ morphological formation in both cancer- and non-tumor-related diseases, in addition to cellular epigenetic transformation and migration, under the influence of LOXs. A number of different signaling pathways regulate the LOXs expression and their enzymatic activation. The tissue remodeling and transformation process shares some resemblance between oncogenesis and embryogenesis. Additionally the roles that LOXs serve appeared to be stressed during oncogenesis and tumor metastasis. It has also been indicated LOXs have a noteworthy role in non-tumor diseases. Nonetheless, the role of LOXs in systemic or local organ development and disease control remains unknown. In the present study, the essential roles that LOXs play in embryogenesis were unveiled partially, whereas the role of LOXs in organ or systematic development requires further investigations. The present review aimed to discuss the roles of members of the LOX family in the context of the remodeling of organogenesis and organ development. In addition, the consequences of the
\end{abstract}

Correspondence to: Professor Jiuhong Yuan, Andrology Laboratory, West China Hospital, Sichuan University, 37 Guoxue Alley, Wuhou, Chengdu, Sichuan 610041, P.R. China

E-mail: jiuhongyuan2107@163.com

*Contributed equally

Abbreviations: BAPN, $\beta$-aminopropionitrile; ECM, extracellular matrix; LOX, lysyl oxidase; LOXL, lysyl oxidase-like proteins; TGF- $\beta$, transforming growth factor- $\beta$

Key words: lysyl oxidase, organogenesis, development, collagen crosslinking, extracellular matrix remodeling malfunction of these proteins related to the development of abnormalities and resulting diseases is discussed.

\section{Contents}

1. Introduction

2. Roles of members of the LOX family in organ development

3. Signaling pathways involved in the modulation of LOX during tissue development

4. Conclusion

\section{Introduction}

Lysyl oxidase proteins (LOXs) are secretory amine oxidases that aid in the formation of the extracellular matrix (ECM) in a copper-dependent manner (1). The LOX precursor (50 kDa), secreted mainly by smooth muscle cells and fibroblasts, is hydrolyzed into the catalytically active, matured form of LOX (30 kDa) and the non-catalytic active peptide (18 kDa) $(2,3)$. The extracellular roles of activated LOX include promoting the crosslinking between ECM collagen type I, collagen type III and elastin, through catalyzing the lysine residue, which subsequently transforms collagens and elastin into a non-soluble state (1).

Since their initial discovery, the role of LOXs in collagen and elastin crosslinking has been confirmed by numerous studies $(1,2,4,5)$, and additional LOX-like proteins (LOXLs)1-4 were subsequently discovered. LOXL1, LOXL2, LOXL3 and LOXL4 have been demonstrated to share $85,58,65$ or $62 \%$ sequence similarity with the LOX protein in the conserved regions and, altogether, these enzymatic proteins form the LOX family (6).

The regulation of LOXs varies depending on the tissue and on the developmental stage of a specific organ; these enzymes have been found to serve a role in oncogenesis or tumor metastasis through dysregulating tumor microenvironment homeostasis and have also been suggested to be biomarkers for cancer prognosis and survival (7). In other studies, LOXs were found to be important in regulating ECM metabolism, which promoted alterations in the tumor tissue tensile strength; consequently, the ECM properties may influence embryogenesis and organ development substantially $(5,7,8)$. Tumorigenesis and embryogenesis involve similar cellular morphological changes, migrations and adjustments in the mechanical stiffness and 
structural integrity of the tissues $(8,9)$. Previous studies have reported contradictory effects of LOXs on the phases of tumor differentiation (5). These contradictions may indicate the spaciotemporal specificities for LOXs response and regulations, which suggest that the response of LOXs may vary at various stages of organ development. A similar phenomenon was observed during embryogenesis, which suggested that LOXs may serve a significant role in the process of ECM structural stabilization and organ development $(10,11)$.

In addition, aberrant ECM metabolism has been identified in several different diseases, including atherosclerosis, liver cirrhosis, aneurysms and Menkes syndrome, and the effects of LOXs were observed to be increased during organogenesis and development (5). ECM remodeling does not only form the foundations for stromal and interstitial stability, it also ensures the proper cellular anchorage to facilitate stabilized cell-cell adhesions, which is essential for maintaining functional stromal polarity (12). In this process, numerous proteins involved in ECM remodeling, including the LOXs, are required to be correctly functioning in the phases of organ development.

\section{Roles of members of the LOX family in organ develop- ment}

The ECM is crucial in regulating extracellular functions. Not only does it maintain tissue mechanical rigidity and stiffness, it is also responsible for manipulating cellular functions and regulating cell migrations $(8,13)$. Amongst the hundreds of components identified in the ECM, fibrous proteins and proteoglycans are the two main constituents $(14,15)$. As these proteins are mainly fibrous in form, the correct crosslinks between these macromolecules are essential for proper tissue development (6,15-18). Furthermore, ECM metabolic processes and remodeling contribute to the complex extracellular mesh, which relies heavily on the catalyzing function of LOXs (19).

During embryogenesis and organ development, LOXs promote infrastructural integration, in addition to facilitating the proper cell stiffness and rigidity required for organogenesis and subsequent morphological maintenance (17,20-23). That is, the cells can be secured and maintained properly in the organic mesh and are able to maintain their functional polarity (12). Owing to the enzymes being secreted locally, the expression levels of LOXs and their subsequent activity have been correlated with the modulation and transformation of the cells. In this manner, cells can detect microenvironmental changes directly or indirectly, and serve as both participants and regulators in response to these changes $(13,24)$. Again, as the mechanical force is becoming increasingly recognized in inducing organ development and tissue remodeling $(25,26)$, further research embryogenesis should not be limited to biochemical factors, but to biomechanical factors as well.

Along with tissue adjustment, cells respond to external stress through the mediation of the ECM, which is also under the influence of the activity of LOXs $(25,27-29)$. In simpler terms, LOXs have been demonstrated to function as mediators between cells and the ECM during development. Thus, this family of enzymes is not only responsible for the overall architecture of cells, but also for the transduction of signals and responses between external forces and parenchymal/stromal cells during the processes of proliferation and remodulation.

Establishment of mechanical strength. The crosslinking of fibrous macromolecules is crucial for tissue formation because the properly formed ECM provides mechanical rigidity and stiffness, which is essential for maintaining the structural correctness and the proper functioning of organs $(8,30,31)$. With regards to the respiratory system, bronchial formation and alveolarization are the main processes that occur during lung development. In a previous study analyzing the expression levels of LOX in the pulmonary parenchyma and pleural membrane during development, it was observed that LOX expression levels in prenatal and postnatal rabbits were increased, and subsequently reduced to $50 \%$ within 4-10 weeks (17). In the same study, a one-side pneumonectomy was performed in hamsters to determine LOX expression levels during the compensatory lung growth; an instant elevation in LOX expression levels were noted, alongside compensatory growth of the lung tissue prior to cellular proliferation. Thus, a chronologic-specific pattern of LOX expression levels was observed during lung growth and development. Conversely, by inhibiting LOX activity, either through downregulating its expression or using the enzymatic inhibitor, $\beta$-aminopropionitrile (BAPN), the newly formed lungs are observed with impaired bronchial morphogenesis and alveolarization $(32,33)$. Notably, Maki et al (20) investigated the $\mathrm{LOX}^{-/-}$and $\mathrm{LOXL1}^{-/}$knockout mice, and it was discovered that the improperly developed airways were associated with abnormally formed elastin and collagen fiber, which resembled the human embryo sample observed in a study by Kumarasamy et al (28). Although the direct evidence that abnormal embryogenesis contributes to pulmonary diseases is lacking, the pulmonary emphysema identified alongside alveolar enlargement and structural distortion were identified to be correlated to ECM abnormalities and LOX downregulation in a previous study (34). Consequently, these findings suggested that the development of the lung mesenchyme, bronchus and the pulmonary artery may be strongly associated with LOX modulation and properly regulated ECM formation may be substantial in maintaining the structural and functional mechanical load of bronchi and alveoli for ventilation and gas exchange.

In $\mathrm{LOX}^{-/-}$mice, it was reported that the mice died perinatally due to aortic aneurysms, cardiovascular dysfunction and diaphragmatic ruptures (20). This result is logical because the structural stability of the cardiovascular system is vital, as it endures the most constant and relative mechanical pressure compared with other organ systems, thus any structural incompetence will lead to fatal consequences. With regards to development, Tsuda et al (35) discovered increased expression levels of LOX mRNA during embryogenic myocardium development in mice on the 11th and 13th day of embryonic development. Similarly, Behmoaras et al (15) revealed that LOX and LOXL1 activities were at their highest during the first 15 days of development, which suggested that both LOXs may be required for elastin and collagen remodeling in the aorta of rats. In addition, the insufficient activity of both enzymes was found to render Brown Norway rats susceptible to spontaneous artery rupture, which further indicated the 
pivotal role of LOXs in ECM regulation, especially in stabilizing collagen and elastin crosslinks $(14,15)$. Complementary research similarly confirmed that LOXs were observed to reside in the aortic arch vessel, amongst other sites as myocardial, endocardial, epicardium (35).

In more concrete organs, such as the teeth or bones, the ECM is found to be mostly mineralized, with other constituents namely being collagen, elastin and other fibrous proteins (18). The development of teeth has been found to involve ECM condensation and odontogenic stabilization (36), of which both processes can be observed through densely packed collagen fibers and orderly polarized picrosirius red staining. Through investigating odontogenesis, Tjaderhane et al (22) identified no significant differences among the teeth of $\mathrm{LOX}^{-/-} \mathrm{LOX}^{+/-}$ and wild-type mice under the light microscope; however, following histochemical examination, the teeth of the $\mathrm{LOX}^{-1-}$ and $\mathrm{LOX}^{+/-}$mice were observed to be thinner and unpolarized, which indicated that these effects may be due to the dysregulation of LOX. Kim et al (37) demonstrated that both LOX and LOXLs were essential for organizing periodontal ECM fibrogenesis and promoting the differentiation of dental pulp cells from odontoblasts. Accordingly, both investigations favor the substantial effect of LOX in promoting the thickening of teeth and matrix collagen filling during development.

Similar to bones, the differentiation and mineralization of stromal cells and ECM are reported to be under LOX regulation. For example, during osteoblastogenesis, a parallel expression pattern between collagen and LOX was found in isolated mice clavicle cells $(38,39)$. This result suggested that LOX may promote osteocyte stabilization and bone ECM remodeling. Furthermore, in $\mathrm{LOX}^{-/}$mice, Pischon et al (40) found reduced osteoblast differentiation and insufficient mineral crystallization. Similarly, Turecek et al (41) inhibited LOX expression with BAPN in cultured osteoblasts and discovered that not only were the collagen crosslinks dysregulated, but the expression and activity of osteoblasts were also undermined. These results further suggested an essential role of LOX in bone matrix formation. The collagen-based mesh weights more crucial than is instinctively comprehend in bones and teeth development; however, the importance of LOXs in regulating development in these organs remains relatively unclear.

The musculoskeletal system accounts for nearly half of the body weight (42). The main constituent of muscles is myofibers, which are coated with muscle connective tissue (MCT) formed from muscle ECM, and muscles and tendons are formed from muscle fibers, fasciculi and other myogenic progenitors (43). Resembles to the ECM in other tissues, the homeostatic metabolism of the MCT is crucial; not only does it provide the supporting forces that bind the muscle fibers together, but the myofiber-MCT cross-talk is crucial during myogenesis (43). Kutchuk et al (44) revealed that muscle fibers formed in $\mathrm{LOX}^{-1-}$ mice were shorter, smaller and decreased in number. Also, the more undeveloped embryonic limbs formed in these mice exhibited a disorganized MCT and the dysregulated deposition of collagen fibrils (44). Thus, it was indicated that insufficient LOX activity may underlie the deformed growth of embryonic limbs, which may be potentially correlated with the origin of Duchenne muscular dystrophy (23).

Attached to the skeletal muscles are the more ECM abundant structures, such as tendons and ligaments. In chick embryos, LOX expression levels in tendons were found to be elevated during their development (26). Moreover, the tendons were found with minor elastic modulus following the application of BAPN during embryogenesis. These results may partially explain the defective healing capability discovered in the anterior cruciate ligament; differential LOX expression levels and collagen crosslinking in the three ligaments of the knee has been found to predispose different healing capabilities $(45,46)$.

The dynamic structural and functional changes during the maturation of the central nervous system (CNS), namely neuronal plasticity, is a critical process of both pre- and postnatal development (47). The involvement of neurogenesis, programmed cell death and ECM remodeling in neuronal plasticity following the adaption to environmental changes is markedly enhanced during brain development (48). In the maturation of the CNS, various components have been identified to be involved in cerebrum and cerebellum ECM remodeling (49). As one of these components, the dendritic extensions of neurons have been observed not only to determine brain function, but also are indicative of a neuron's development and its regeneration. A previous study identified the presence of LOXs in the cerebrum and immunohistochemical analysis further revealed that cells in the pyramid layer in the hippocampus of LOXL-null mice exhibited decreased diameters (50). These results revealed a potential essential role of LOXLs in inducing cellular differentiation; for example, it has been discovered that the increase in intranuclear LOX-propeptide facilitated microtubule stability and dendritic cell development (51).

Similarly, as the genetic deficiency of vacuolar protein sorting protein 18 (VPS18) is reported to facilitate the lysosomal degradation of LOX, the impaired dendritogenesis in VPS18 knockout mice was suggested to be correlated with the accumulation of LOX (52). That study revealed that other than ECM remodeling, LOX may also be capable of regulating organ development. Additionally, in studying amputated mice, the white matter and gray matter in the spinal cord were both found to be atrophied (53). Meanwhile, the decreased myelination and downregulated ECM regulatory factors were depressed along with LOX expression levels in another study (53). Furthermore, in superoxide dismutase 1-induced neurodegenerative model rats, neurons in the developed amyotrophic lateral sclerosis were found to express increased LOX expression levels and exhibit increased enzyme activity (54). This evidence suggested that, as the principle form of ECM remodulation in the CNS, synaptic remodeling may be regulated by both LOX and neuronal signaling transmissions. Thus, proper regulation with a spatial and conditional specialty is fundamental for CNS development.

In a previous study, LOXs were found widely distributed cutaneously and subcutaneously, and were discovered to be correlated with aging (55). In fact, LOX expression was abundant in the epidermal basal layer, the basal keratinocytes and dermal fibroblasts, dermal vascular endothelial cells, hair follicles, sebaceous glands, sweat glands and hair $(55,56)$. Cenizo et al (57) demonstrated that LOX expression levels in skin fibroblasts in adults were decreased compared with children, whereas, Langton et al (58) identified higher LOX activity in the elderly compared with young people. 
In epidermal keratinocytes, further research identified an important role for LOX in regulating cellular keratinization, whereas LOXL2 was found to interact with cell-matrix interactions (59-61). Le Provost et al (60) have thus suggested that finely regulated LOX expression is crucial for maintaining epidermal homeostasis.

LOX mediates the ECM response to environmental change. During organogenesis, the interaction between cells and the ECM in the adapting environment change is crucial (8). Physiological and biochemical changes in the environment, namely hypoxia, glycation, hormonal changes and deposition of metabolites, were discovered to trigger alterations in ECM formation and enzyme expression (17,62-65). These adaptations in turn required the responses of the stroma and ECM to initiate the post-translational tissue remodeling process $(7,8,13)$.

Pneumonectomy and hypoxia treatment are the conventional methods used to investigating tissue alterations during lung organogenesis (17). As an ECM modulating enzyme, it was observed that hypoxia triggered cellular responses directly through increasing the expression levels of LOX (66). Cells are known to be sensitive to and promptly react to tissue oxygen saturation $\left(\mathrm{StO}_{2}\right)$, as the $\mathrm{O}_{2}$ in the ECM is directly exchanged through passive diffusion extra-intracellularly (67). Low $\mathrm{StO}_{2}$ directly hinders hypoxia induced factors (the HIF) from degrading, hence its accumulation triggers subsequent signaling cascades in response to hypoxia (68). In fact, low $\mathrm{O}_{2}$ levels were ubiquitously observed to increase LOX expression levels and mediate ECM remodeling among different organogenesis and tissue development $(29,35,66,69)$. In a model of pulmonary arterial hypertension, it was demonstrated that the vascular smooth muscle cells in the pulmonary arteries responded to hypoxia through increased LOX expression levels and subsequent enzymatic activity $(64,70)$. Furthermore, this contributed to the increased deposition of collagen and elastin, alongside enhanced cellular proliferation during the progression of pulmonary vascular remodeling. Further studies have also identified the participation of other LOXLs, such as LoxL1, LoxL2, LoxL3 and LoxL4, contributing to idiopathic pulmonary arterial hypertension with response to hypoxia (71). Although this was observed in a pathological model, a similar mechanism is presumably employed during tissue development. Similarly, LOX-mediated ECM remodeling in response to hypoxia has been reported in myocardial ischemia, liver fibrosis and in patients with obstructive sleep apnea $(72,73)$. In addition, through investigating tendon and cartilage structure, the tenocytes were found to respond to hypoxia following increased LOX expression levels $(29,74,75)$; as a consequence, these tenocytes were found with enhanced expansion capacity and proliferative potential. These results demonstrated that monitoring LOX expression, as the direct cellular response to hypoxia, is potentially useful as a biomarker and as a tissue engineering target to control tissue development.

Similar to hypoxia, dysregulated glycation induced tissue pathophysiology with altered ECM formation is a feature of metabolic diseases (76). It is reported that collagen crosslinking pathways involve both non-enzymatic glycation and LOX-mediated oxidative deamination of lysine and hydroxylysine (77). Commonly, dysregulated glycation is a result of diabetes, which has been indicated to induce altered enzymatic crosslinks and collagen physicochemical properties of the ECM in multiple systems (76). In the skin, patients with diabetes are more likely to appear aged and have a skin infection or foot ulcers, which is accompanied by fragmented collagens and dysregulated dermal connective tissues (78). In the retina, diabetes resulted in structural abnormalities in the retinopathy, which featured as thickened retinal capillary basement membranes with upregulated levels of ECM fibroproteins $(79,80)$. These findings may seem contradictory. A plausible explanation for this phenomenon is that the structural changes in the oculus tissue were the consequence of excessive microvasculature permeability, whereas the abnormal collagen crosslinking change was similar to other hyperglycemia developed lesions (77). These results indicated that the glycation that contributes to ECM formation through LOX may be regulated at the metabolic level, as well as at the vascular and oxidative levels. For example, high glucose levels were discovered to directly increase the expression levels of LOX and its subsequent enzymatic activation in the dermis or endothelial layer, which resulted in excessive crosslinking and disruption in the formation of collagen fibrils and hindered ECM integrity (80). Furthermore, increased expression levels of matrix metalloproteinase (MMP)-1 and MMP-2 were identified in glycation-induced LOX dysregulation; this result revealed the existence of indirect regulation between LOX and glycation (78).

Native low-density lipoprotein and alcohol have also been reported to reduce LOX expression, collagen and elastin crosslinking in endothelial cells and the formation of scar tissue $(65,81)$. Though these results were obtained following pathogenic studies, the implication of these results in manipulating organogenesis requires further investigations.

Similarly, in age-related macular degeneration (AMD), pathological alterations involve choroidal neovascularization, choroidal capillary proliferation and aberrant basement membrane architecture (82). Upon investigating environmental tobacco smoke-induced AMD, it was observed that the side stream smoke may directly suppress LOX expression in choroidal endothelial cells (83).

Thus, ECM formation and its regulation are complex issues involving complex mechanisms in developing tissues. In those organs or systems in which their structural or mechanical properties mainly determine the function, the appropriate regulation of the formation of the ECM is substantial for the mechanical strength required for structural maintenance and tension upholding, but also in providing the foundations for initiating growth. Nonetheless, studies have also revealed that LOXs are able to manipulate the transformation of the cellular phenotype in response to environmental changes during organogenesis $(35,37,38,84)$. These results suggested that the role of LOXs in regulating organ development remain to be fully determined (see also Table I).

\section{Signaling pathways involved in the modulation of LOX during tissue development}

Numerous factors have been identified that regulate LOX; for example, the stimulation of fibroblasts or myofibroblasts prompts the secretion of the LOX precursor or promotes LOX 
Table I. Role of LOX and LOXL in organ development.

\begin{tabular}{|c|c|c|c|c|}
\hline Authors, year & Tissue & Member & Role & Refs. \\
\hline Rauch, 2004 & Cerebrum & LOX & Elevated in superoxide dismutase 1 -induced neurodegeneration & $(49)$ \\
\hline Li et al, 2010 & & LOX-pro-peptide & Interfered with NF- $\kappa \mathrm{B}$ RelA signaling and microtubule stability & $(51)$ \\
\hline Peng et al, 2012 & & LOX & $\begin{array}{l}\text { VPS } 18 \text { gene knockdown impairs dendritogenesis following the } \\
\text { accumulation of LOX }\end{array}$ & $(52)$ \\
\hline Chelyshev et al, 2014 & Spinal cord & LOX & Decreased expression alongside decreased myelination & $(53)$ \\
\hline Brody et al, 1979 & Lung & LOX & $\begin{array}{l}\text { Newborn rabbit exhibited transient increased expression levels } \\
\text { compared with adult rabbits, and were reduced within } \\
\text { 4-10 weeks after birth }\end{array}$ & $(17)$ \\
\hline Tsuda et al, 2003 & Heart & LOX & $\begin{array}{l}\text { Positively correlated with embryogenic myocardium } \\
\text { development }\end{array}$ & $(35)$ \\
\hline Hornstra et al, 2003 & Aorta & LOX & $\begin{array}{l}\text { Genetic deletion in mice caused aneurysms and diaphragmatic } \\
\text { rupture }\end{array}$ & (14) \\
\hline Voloshenyuk et al, 2011 & & LOX & Facilitated vascular ECM hardening and remodeling & $(94)$ \\
\hline Tjaderhane et al, 2013 & Tooth & LOX and LOXLs & $\begin{array}{l}\text { Promoted pulp medulla dentinal cellular differentiation, ECM } \\
\text { augmentation and mineral nodule formation }\end{array}$ & $(22)$ \\
\hline Kaku et al, 2016 & & LOX & $\begin{array}{l}\text { Responded to mechanical stress and promoted odontogenic } \\
\text { differentiation }\end{array}$ & $(36)$ \\
\hline Tjaderhane et al, 2013 & & LOX & Promoted tooth thickening and matrix collagen filling & $(22)$ \\
\hline Vora et al, 2010 & Bone & LOX and LOXLs & $\begin{array}{l}\text { Promoted osteoblast differentiation and bone matrix } \\
\text { mineralization }\end{array}$ & $(39)$ \\
\hline Pischon et al, 2009 & & LOX & $\begin{array}{l}\beta \text {-aminopropionitrile or genetic knockout reduced osteoblast } \\
\text { differentiation and osteoblast deactivation }\end{array}$ & $(40)$ \\
\hline Vora et al, 2010 & & LOX-pro-peptide & Inhibited osteoblast proliferation and differentiation & $(39)$ \\
\hline Makris et al, 2013 & Cartilage & LOX & Strengthened cartilage by hypoxia induction & $(29)$ \\
\hline Marturano et al, 2014 & Muscle and & LOX & Promoted collagen fibril formation in muscle and tendons & $(74)$ \\
\hline $\begin{array}{l}\text { Xie et al, } 2012 \\
\text { Kato } \text { et al, } 2015\end{array}$ & tendon & LOX & Promote collagen maturation in cruciate ligament & $\begin{array}{l}(45) \\
(46)\end{array}$ \\
\hline Szauter et al, 2005 & Skin & LOX and LOXL2 & $\begin{array}{l}\text { Deactivated with aging, dynamically expressed by fibroblasts } \\
\text { with the cellular response }\end{array}$ & $(55)$ \\
\hline Jiang et al, 2014 & Uterus & LOX & Maintained by estrogen and downregulated during aging & $(62)$ \\
\hline
\end{tabular}

LOX, lysyl oxidase; ECM, extracellular matrix; LOXL, LOX-like proteins; VPS18, vacuolar protein sorting protein 18.

hydroxylation and direct enzymatic activation (30). It was reported that hypoxia-inducible factor (HIF) $1 \alpha$, advanced glycation end-products-dependent transcription factor, transforming growth factor (TGF)- $\beta$, tolloid protein-1 (TLD1) and fibronectin all promoted either the expression or activation of LOX (33,84-89). The opposite effect occurred following the stimulation from prostaglandin E2 and homocysteine $(11,16)$. BAPN is extensively applied in in vivo and in vitro experiments as it selectively and non-reversibly inhibits LOX activity (5).

During the branching of airways and pulmonary vasculogenesis, hypoxia is one of the deciding promoters that triggers their development $(66,86)$. Therefore, hypoxia treatment or lateral-pneumonectomy are the most common animal model methods used for studying the issues of the respiratory system (17). In tissues with high expression levels of HIF1 $\alpha$ and HIF2, the tissue exhibited increased fibrogenesis and collagen deposition, alongside increased expression levels of
LOX (69). Decreased HIF expression levels were found to lead to vascular alveolar hypoplasia, neonatal respiratory distress and bronchopulmonary dysplasia that featured alongside insufficiently deposited and crosslinked collagens (66). It is therefore suggested that LOX may serve an important role in ECM modeling under HIF regulation, as Pez et al (90) revealed that HIF and LOX synergistically promote the growth of newly formed tissue and increased cellular proliferation in these tissues.

Fibrosis serves an important role in structural reconfiguration during development. Although LOX and LOXLs are reported to significantly promote fibrotic alterations in tissues, certain stimuli are required to stimulate fibroblasts or myofibroblasts to secrete their precursors $(91,92)$. In addition, the increased hydroxylation of LOX and its direct enzymatic activation are required (30). The presence of TGF- $\beta$ is required to facilitate the growth of embryos and fetal myoblast fibroblasts; 


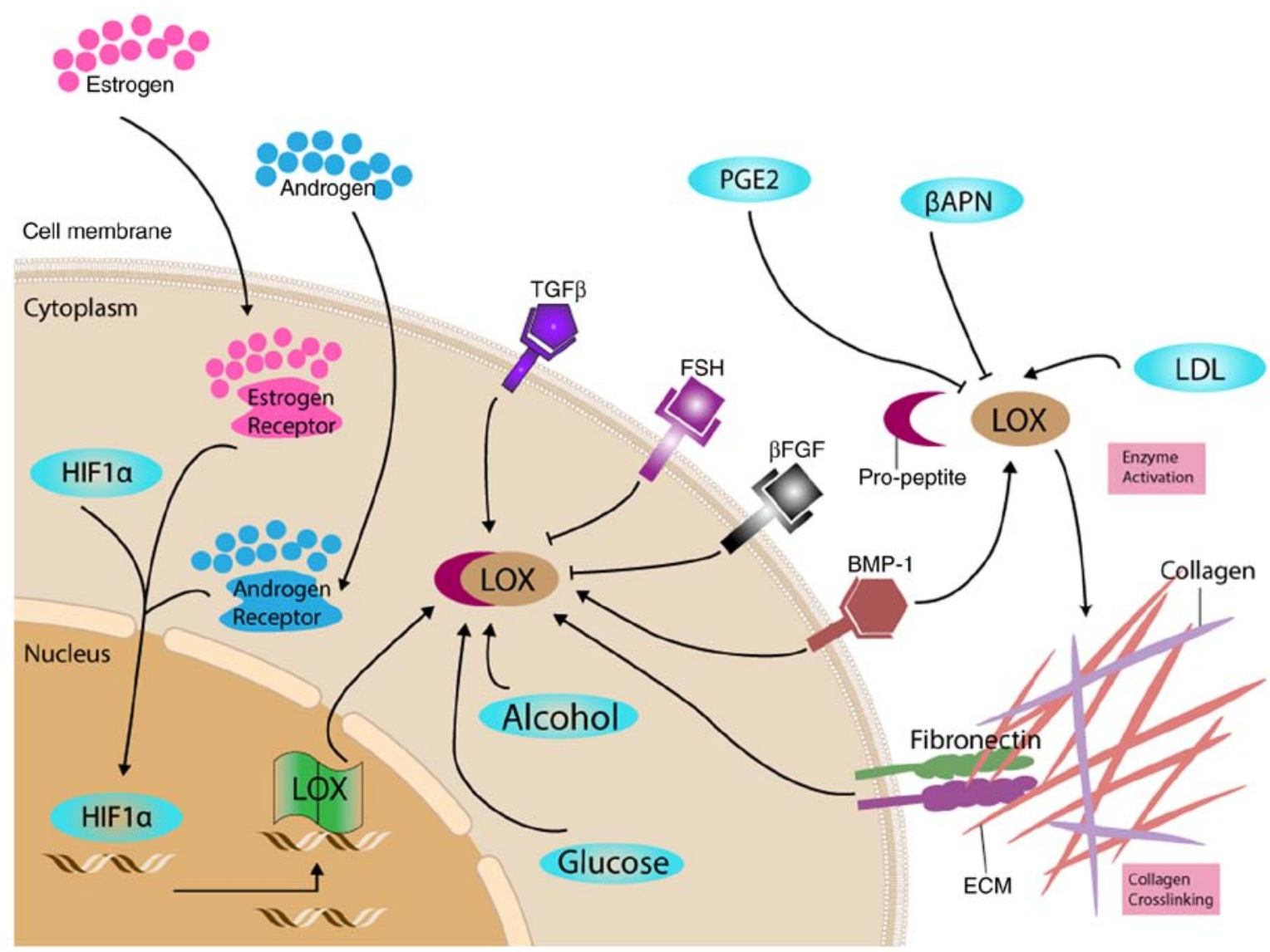

Figure 1. Proposed roles of LOX in response to different factors. LOX was expressed and secreted in its proenzyme form. The regulation of LOX occurs at multiple levels, including at the transcriptional and translational level, and following both intracellular and extracellular enzyme activation. These regulations and reactions are correlated with the ECM transformation during embryogenesis and leads to changes in cell adhesion and proliferation, which correspond to organ development. $\beta$ APN, $\beta$-aminopropionitrile; $\beta \mathrm{FGF}$, fibroblast growth factor $\beta$; BMP-1, bone morphogenetic protein-1; ECM, extracellular matrix; FSH, follicle-stimulating hormone; HIF1 $\alpha$, hypoxia-inducible factor $1 \alpha$; LDL, low density lipoprotein; LOX, lysyl oxidase; TGF- $\beta 1$, transforming growth factor- $\beta 1$; PGE2, prostaglandin E2.

Cusella-De Angelis et al (85) and Leonard et al (93) both discovered that TGF- $\beta$ triggered the proliferation of fetal myoblasts and fibroblasts. This may in turn stimulate primary limb bud formation, which is essential for the secondary level of limb development, and ECM and stromal enrichment. In addition, in neonatal rat aorta smooth muscle cells, TGF- $\beta 1$ was found to significantly promote LOX expression levels (35). A similar result was reported in cardiac fibroblasts treated with TGF- $\beta$; it was observed that LOX expression levels were increased at both the mRNA and protein level, and this may be prevented by inhibitors of the TGF- $\beta$ cascade (94). These findings suggested that the presence of TGF- $\beta$ may be essential for maintaining LOX stability.

Within in the TGF- $\beta$ superfamily, the bone morphogenetic proteins (BMPs) have also been demonstrated to influence organogenesis in the brain, eye, hair follicles, kidney, lung, liver, skin and teeth in a pleiotropic manner (89). In regulating the morphogenesis of each organ, BMP was found to activate LOX and LOXL1 from pro-enzymes, thus it can be suggested that they are both critical for the ECM crosslinking that determines the biomechanical features of the tissue (95). Additionally, BMP-1 was found to promote the efficiency of pro-LOX activity between 1- and 20-fold compared with mammalian TLD (mTLD) or mammalian TLD-like (mTLL)-1 and mTLL-2 (89). Thus, it was suggested that BMP may regulate embryogenic fibroblasts and control LOX activity in an mTLD and MTTD assisted manner (96).

Similar to the crosslinking between collagens, the elastin-collagen crosslinking determines the stability of the ECM $(15,84)$. Elastin stabilization requires the catalyzation of LOX, whereas, elastin and collagen crosslinking require the catalyzation of fibronectins (FNs), a type of glycoprotein that is abundantly expressed in the ECM (88). FN receptors are located in every tissue that originates from the three primary germ layers and are therefore considered to modulate embryogenic cellular migration and anchoring (24), which suggested the FN signaling may mediate the cellular-ECM signal transduction that may, in turn, modulate LOX secretion. Fogelgren et al (97) revealed that the decreased LOX proteolytic processing in FN-null mouse embryonic fibroblasts was associated with lower LOX activity; however, whilst their enzymatic activity data did not determine the role of cellular FN (cFN) in regulating LOX, it was hypothesized that the FN matrix may activate LOX in a comprehensive manner. Specifically, FN was hypothesized to be expressed differently in various stages of organ development with the alteration of tissue microenvironment.

In LOXL1 knockout mice, the malformed pelvic floor, weakened vaginal wall and tendencies of pelvic organ prolapse were observed (98). A similar phenomenon was also observed in estrogen-deficient mice (63). Furthermore, it was noticed 
that estrogen promoted LOX upregulation and enzymatic maturation through direct and indirect means, by which such a mechanism is deemed beneficial in maintaining pelvic wall stability and skin elasticity $(63,99)$. Notably, the pro-LOX effect of estrogen was found to be inhibited following the administration of SB431542, a TGF- $\beta 1$ receptor inhibitor, revealing the participation of TGF- $\beta$ signaling in the estrogenic effect (63). Similarly, the pro-LOX effect was also identified with androgens, as Harlow et al (100) and Slee et al (101) identified that $5 \alpha$-dihydrotestosterone significantly increased LOX expression levels both in vitro and in vivo. In addition, the follicle-stimulating hormone was observed to reduce both LOX expression levels and its activity (100). These results revealed the complicated regulation of LOX by the sex hormones and indicated a potential target in manipulating organogenesis through LOX regulation.

Multiple signaling pathways are involved in LOX and LOXLs regulation at different levels, including pathways involved in genetic transcription, enzymatic synthesis and activation both intracellularly and extracellularly. ECM maturation facilitates cellular transformation, which results in embryogenic progression and organ development. Fibroblasts and SMCs are both considered as primary regulators and messengers. For they were observed not only receiving shifting signaling and extracellular feedbacks (91). In turn, they stimulate alterations that contribute to the stromal changes and tissue biomechanical alterations (such as changes in the rigidity) in response to organ-specific needs and parenchymal requirements (33). The response of LOX to multiple factors in organ development is summarized in Fig. 1.

However, how to utilize these signaling effects and the ECM-cellular responses described above to manipulate ECM formation and organogenesis with LOXs requires further investigation; the prevention of abnormal development through targeting LOX requires a more thorough understanding of the regulation of LOXs and their spatiotemporal specificity, which currently remains relatively unknown.

\section{Conclusion}

ECM metabolism serves a critical role in tissue development; not only is it an essential mechanical structure for cells to maintain normal organ function and transducing extracellular mechanical signals to stimulate cellular responses, but it also forms the microenvironment that enables stromal and parenchymal interactions. Apart from triggering inter-collagen crosslinking that determines tissue stiffness, LOXs are demonstrated to be involved in multiple physiological or pathological pathways, both in extracellular modulation and intracellular signaling. The findings of the present review suggested that LOX may be comprehensively involved in the organogenesis of all systems. Nevertheless, regulating mechanical homeostasis should be considered as the pivotal role of LOX.

In order to decipher this pivotal role, efforts should focus on investigating how LOXs modulates the ECM by maintaining the mechanical properties, which are essential in maintaining organ integrity and organ functional properties. During this process, direct and indirect influences of LOX on the stromal and parenchymal interactions that guide cellular phenotypic adaptations is also worth of further investigation.
A number of methods have proven effective in targeting LOX; however, research remains far from declaring a plausible and credible method for regulating LOX expression. The complexity and spatiotemporal specificity of LOX requires further investigations, followed by its modulatory role in physiological and pathophysiological states. Studies on LOXs have been conducted for decades; however, there are currently no clinical trials on LOXs for disease prevention and treatment, which suggested that the current understanding of LOXs is primitive. Thus, the application of LOX genes and proteins on disease diagnosis, treatment and prognosis are required to be further investigated.

\section{Acknowledgements}

Not applicable.

\section{Funding}

This study was supported by the Natural Science Foundation of China (grant nos. 81671453, 81270691, and 81170565), Science and Technology Project of the Health Planning Committee of Sichuan (grant no. 18PJ487) and Thousand Talent Plan of Sichuan Province.

\section{Availability of data and materials}

Not applicable.

\section{Authors' contributions}

LG, JY, FQ and CW performed the conception and design of the study. JY and FQ provided administrative support. SW, LG and JY aided with the provision of study materials or patients. LG, JY, SW and CW collected and assembled the data. SW, LG, YW and CW performed the data analysis and interpretation. SW, LG, JY and CW wrote the manuscript. All authors read and approved the final manuscript.

\section{Ethics approval and consent to participate}

Not applicable.

\section{Patient consent for publication}

Not applicable.

\section{Competing interests}

The authors declare that they have no competing interests.

\section{References}

1. Finney J, Moon HJ, Ronnebaum T, Lantz M and Mure M: Human copper-dependent amine oxidases. Arch Biochem Biophys 546: 19-32, 2014.

2. Lucero HA and Kagan HM: Lysyl oxidase: An oxidative enzyme and effector of cell function. Cell Mol Life Sci 63: 2304-2316, 2006.

3. Papadantonakis N, Matsuura S and Ravid K: Megakaryocyte pathology and bone marrow fibrosis: The lysyl oxidase connection. Blood 120: 1774-1781, 2012. 
4. Smith-Mungo LI and Kagan HM: Lysyl oxidase: Properties, regulation and multiple functions in biology. Matrix Biol 16: 387-398, 1998

5. Barker HE, Cox TR and Erler JT: The rationale for targeting the LOX family in cancer. Nat Rev Cancer 12: 540-552, 2012.

6. Chen L, Li S and Li W: LOX/LOXL in pulmonary fibrosis: Potential therapeutic targets. J Drug Target 27: 790-796, 2019.

7. Amendola PG, Reuten R and Erler JT: Interplay Between LOX Enzymes and Integrins in the Tumor Microenvironment. Cancers (Basel) 11: 11, 2019.

8. Nieto MA, Huang RY, Jackson RA and Thiery JP: Emt: 2016. Cell 166: 21-45, 2016.

9. Dongre A and Weinberg RA: New insights into the mechanisms of epithelial-mesenchymal transition and implications for cancer. Nat Rev Mol Cell Biol 20: 69-84, 2019.

10. Polgar N, Fogelgren B, Shipley JM and Csiszar K: Lysyl oxidase interacts with hormone placental lactogen and synergistically promotes breast epithelial cell proliferation and migration. J Biol Chem 282: 3262-3272, 2007.

11. Rosenbloom J, Ren S and Macarak E: New frontiers in fibrotic disease therapies: The focus of the Joan and Joel Rosenbloom Center for Fibrotic Diseases at Thomas Jefferson University. Matrix Biol 51: 14-25, 2016.

12. Lewis PL, Yan M, Su J and Shah RN: Directing the growth and alignment of biliary epithelium within extracellular matrix hydrogels. Acta Biomater 85: 84-93, 2019.

13. Al Ameri W, Ahmed I, Al-Dasim FM, Ali Mohamoud Y, Al-Azwani IK, Malek JA and Karedath T: Cell Type-Specific TGF- $\beta$ Mediated EMT in 3D and 2D Models and Its Reversal by TGF- $\beta$ Receptor Kinase Inhibitor in Ovarian Cancer Cell Lines. Int J Mol Sci 20: 20, 2019.

14. Hornstra IK, Birge S, Starcher B, Bailey AJ, Mecham RP and Shapiro SD: Lysyl oxidase is required for vascular and diaphragmatic development in mice. J Biol Chem 278: 14387-14393, 2003.

15. Behmoaras J, Slove S, Seve S, Vranckx R, Sommer P and Jacob MP: Differential expression of lysyl oxidases LOXL1 and LOX during growth and aging suggests specific roles in elastin and collagen fiber remodeling in rat aorta. Rejuvenation Res 11: 883-889, 2008

16. Tang SS, Trackman PC and Kagan HM: Reaction of aortic lysyl oxidase with beta-aminopropionitrile. J Biol Chem 258 4331-4338, 1983

17. Brody JS, Kagan H and Manalo A: Lung lysyl oxidase activity: Relation to lung growth. Am Rev Respir Dis 120: 1289-1295, 1979.

18. Mammoto T, Mammoto A, Jiang A, Jiang E, Hashmi B and Ingber DE: Mesenchymal condensation-dependent accumulation of collagen VI stabilizes organ-specific cell fates during embryonic tooth formation. Dev Dyn 244: 713-723, 2015.

19. Gartland A, Erler JT and Cox TR: The role of lysyl oxidase, the extracellular matrix and the pre-metastatic niche in bone metastasis. J Bone Oncol 5: 100-103, 2016.

20. Mäki JM, Sormunen R, Lippo S, Kaarteenaho-Wiik R, Soininen R and Myllyharju J: Lysyl oxidase is essential for normal development and function of the respiratory system and for the integrity of elastic and collagen fibers in various tissues. Am J Pathol 167: 927-936, 2005.

21. Eberson LS, Sanchez PA, Majeed BA, Tawinwung S, Secomb TW and Larson DF: Effect of lysyl oxidase inhibition on angiotensin II-induced arterial hypertension, remodeling, and stiffness. PLoS One 10: e0124013, 2015.

22. Tjäderhane L, Vered M, Pääkkönen V, Peteri A, Mäki JM, Myllyharju J, Dayan D and Salo T: The expression and role of Lysyl oxidase (LOX) in dentinogenesis. Int Endod J 46: 581-589, 2013.

23. Heinemeier KM, Olesen JL, Haddad F, Langberg H, Kjaer M, Baldwin KM and Schjerling P: Expression of collagen and related growth factors in rat tendon and skeletal muscle in response to specific contraction types. J Physiol 582: 1303-1316, 2007.

24. Duband JL, Rocher S, Chen WT, Yamada KM and Thiery JP Cell adhesion and migration in the early vertebrate embryo: Location and possible role of the putative fibronectin receptor complex. J Cell Biol 102: 160-178, 1986.

25. Athanasiou KA, Responte DJ, Brown WE and Hu JC: Harnessing biomechanics to develop cartilage regeneration strategies. J Biomech Eng 137: 020901, 2015.

26. Pan XS, Li J, Brown EB and Kuo CK: Embryo movements regulate tendon mechanical property development. Philos Trans R Soc Lond B Biol Sci 373: 373, 2018.
27. Adam O, Theobald K, Lavall D, Grube M, Kroemer HK, Ameling S, Schäfers HJ, Böhm M and Laufs U: Increased lysyl oxidase expression and collagen cross-linking during atrial fibrillation. J Mol Cell Cardiol 50: 678-685, 2011.

28. Kumarasamy A, Schmitt I, Nave AH, Reiss I, van der Horst I, Dony E, Roberts JD Jr, de Krijger RR, Tibboel D, Seeger W, et al: Lysyl oxidase activity is dysregulated during impaired alveolarization of mouse and human lungs. Am J Respir Crit Care Med 180: 1239-1252, 2009.

29. Makris EA, Hu JC and Athanasiou KA: Hypoxia-induced collagen crosslinking as a mechanism for enhancing mechanical properties of engineered articular cartilage. Osteoarthritis Cartilage 21: 634-641, 2013.

30. López B, González A, Hermida N, Valencia F, de Teresa E and Díez J: Role of lysyl oxidase in myocardial fibrosis: From basic science to clinical aspects. Am J Physiol Heart Circ Physiol 299: H1-H9, 2010.

31. Magnusson SP, Heinemeier KM and Kjaer M: Collagen Homeostasis and Metabolism. Adv Exp Med Biol 920: 11-25, 2016.

32. Mammoto T, Jiang E, Jiang A and Mammoto A: Extracellular matrix structure and tissue stiffness control postnatal lung development through the lipoprotein receptor-related protein 5/Tie2 signaling system. Am J Respir Cell Mol Biol 49: 1009-1018, 2013.

33. Hashimoto S, Nakano H, Suguta Y, Irie S, Jianhua L and Katyal SL: Exogenous fibroblast growth factor-10 induces cystic lung development with altered target gene expression in the presence of heparin in cultures of embryonic rat lung. Pathobiology 79: 127-143, 2012.

34. Besiktepe N, Kayalar O, Ersen E and Oztay F: The copper dependent-lysyl oxidases contribute to the pathogenesis of pulmonary emphysema in chronic obstructive pulmonary disease patients. J Trace Elem Med Biol 44: 247-255, 2017.

35. Tsuda T, Pan TC, Evangelisti L and Chu ML: Prominent expression of lysyl oxidase during mouse embryonic cardiovascular development. Anat Rec A Discov Mol Cell Evol Biol 270: 93-96, 2003.

36. Kaku M, Rosales Rocabado JM, Kitami M, Ida T, Akiba Y, Yamauchi M and Uoshima K: Mechanical Loading Stimulates Expression of Collagen Cross-Linking Associated Enzymes in Periodontal Ligament. J Cell Physiol 231: 926-933, 2016.

37. Kim EC, Lee HJ and Kim Y: Lysyl oxidase and the lysyl oxidase-like protein modulate odontoblastic differentiation of human dental pulp cells. J Endod 38: 769-773, 2012.

38. Sharma-Bhandari A, Park SH, Kim JY, Oh J and Kim Y: Lysyl oxidase modulates the osteoblast differentiation of primary mouse calvaria cells. Int J Mol Med 36: 1664-1670, 2015.

39. Vora SR, Palamakumbura AH, Mitsi M, Guo Y, Pischon N, Nugent MA and Trackman PC: Lysyl oxidase propeptide inhibits FGF-2-induced signaling and proliferation of osteoblasts. J Biol Chem 285: 7384-7393, 2010.

40. Pischon N, Mäki JM, Weisshaupt P, Heng N, Palamakumbura AH, N'Guessan P, Ding A, Radlanski R, Renz H, Bronckers TA, et al: Lysyl oxidase (lox) gene deficiency affects osteoblastic phenotype. Calcif Tissue Int 85: 119-126, 2009.

41. Turecek C, Fratzl-Zelman N, Rumpler M, Buchinger B, Spitzer S, Zoehrer R, Durchschlag E, Klaushofer K, Paschalis EP and Varga F: Collagen cross-linking influences osteoblastic differentiation. Calcif Tissue Int 82: 392-400, 2008.

42. Janssen I, Heymsfield SB, Wang ZM and Ross R: Skeletal muscle mass and distribution in 468 men and women aged 18-88 yr. J Appl Physiol 89: 81-88, 2000.

43. Hasson P: 'Soft' tissue patterning: Muscles and tendons of the limb take their form. Dev Dyn 240: 1100-1107, 2011

44. Kutchuk L, Laitala A, Soueid-Bomgarten S, Shentzer P, Rosendahl AH, Eilot S, Grossman M, Sagi I, Sormunen R, Myllyharju J, et al: Muscle composition is regulated by a Lox-TGF $\beta$ feedback loop. Development 142: 983-993, 2015.

45. Xie J, Jiang J, Zhang Y, Xu C, Yin L, Wang C, Chen PC and Sung KL: Up-regulation expressions of lysyl oxidase family in Anterior Cruciate Ligament and Medial Collateral Ligament fibroblasts induced by Transforming Growth Factor-Beta 1. Int Orthop 36: 207-213, 2012

46. Kato S, Saito M, Funasaki H and Marumo K: Distinctive collagen maturation process in fibroblasts derived from rabbit anterior cruciate ligament, medial collateral ligament, and patellar tendon in vitro. Knee Surg Sports Traumatol Arthrosc 23: 1384-1392, 2015.

47. Takesian AE and Hensch TK: Balancing plasticity/stability across brain development. Prog Brain Res 207: 3-34, 2013. 
48. Ismail FY, Fatemi A and Johnston MV: Cerebral plasticity: Windows of opportunity in the developing brain. Eur J Paediatr Neurol 21: 23-48, 2017.

49. Rauch U: Extracellular matrix components associated with remodeling processes in brain. Cell Mol Life Sci 61: 2031-2045, 2004.

50. Bronson NW, Hamilton JS, Han M, Li PA, Hornstra I, Horowitz JM and Horwitz BA: LOXL null mice demonstrate selective dentate structural changes but maintain dentate granule cell and CA1 pyramidal cell potentiation in the hippocampus. Neurosci Lett 390: 118-122, 2005.

51. Li J, Gu X, Ma Y, Calicchio ML, Kong D, Teng YD, Yu L, Crain AM, Vartanian TK, Pasqualini R, et al: Nna1 mediates Purkinje cell dendritic development via lysyl oxidase propeptide and NF- $\kappa$ B signaling. Neuron 68: 45-60, 2010.

52. Peng C, Yan S, Ye J, Shen L, Xu T and Tao W: Vps18 deficiency inhibits dendritogenesis in Purkinje cells by blocking the lysosomal degradation of Lysyl Oxidase. Biochem Biophys Res Commun 423: 715-720, 2012

53. Chelyshev YA, Muhamedshina YO, Povysheva TV, Shaymardanova GF, Rizvanov AA, Nigmetzyanova MV, Tiapkina OV, Bondarenko NI, Nikolskiy EE and Islamov RR: Characterization of spinal cord glial cells in a model of hindlimb unloading in mice. Neuroscience 280: 328-339, 2014.

54. Li PA, He Q, Cao T, Yong G, Szauter KM, Fong KS, Karlsson J, Keep MF and Csiszar K: Up-regulation and altered distribution of lysyl oxidase in the central nervous system of mutant SOD1 transgenic mouse model of amyotrophic lateral sclerosis. Brain Res Mol Brain Res 120: 115-122, 2004.

55. Szauter KM, Cao T, Boyd CD and Csiszar K: Lysyl oxidase in development, aging and pathologies of the skin. Pathol Biol (Paris) 53: 448-456, 2005.

56. Yamazaki Y, Mikami Y, Yuguchi M, Namba Y and Isokawa K: Development of collagen fibres and lysyl oxidase expression in the presumptive dermis of chick limb bud. Anat Histol Embryol 41: 68-74, 2012.

57. Cenizo V, André V, Reymermier C, Sommer P, Damour O and Perrier E: LOXL as a target to increase the elastin content in adult skin: A dill extract induces the LOXL gene expression. Exp Dermatol 15: 574-581,2006.

58. Langton AK, Griffiths CE, Sherratt MJ and Watson RE: Cross-linking of structural proteins in ageing skin: An in situ assay for the detection of amine oxidase activity. Biogerontology 14 89-97, 2013.

59. Fujimoto E and Tajima S: Reciprocal regulation of LOX and LOXL2 expression during cell adhesion and terminal differentiation in epidermal keratinocytes. J Dermatol Sci 55: 91-98, 2009

60. Le Provost GS, Debret R, Cenizo V, Aimond G, Pez F, Kaniewski B, André V and Sommer P: Lysyl oxidase silencing impairs keratinocyte differentiation in a reconstructed-epidermis model. Exp Dermatol 19: 1080-1087, 2010.

61. Noblesse E, Cenizo V, Bouez C, Borel A, Gleyzal C, Peyrol S Jacob MP, Sommer P and Damour O: Lysyl oxidase-like and lysyl oxidase are present in the dermis and epidermis of a skin equivalent and in human skin and are associated to elastic fibers. J Invest Dermatol 122: 621-630, 2004

62. Jiang Y, Zong W, Luan H, Liu JH, Zhang AZ, Li XL, Liu SY, Zhang SQ and Gao JG: Decreased expression of elastin and lysyl oxidase family genes in urogenital tissues of aging mice. J Obstet Gynaecol Res 40: 1998-2004, 2014.

63. Zong W, Jiang Y, Zhao J, Zhang J and Gao JG: Estradiol plays a role in regulating the expression of lysyl oxidase family genes in mouse urogenital tissues and human Ishikawa cells. J Zhejiang Univ Sci B 16: 857-864, 2015.

64. Zimnicka AM, Tang H, Guo Q, Kuhr FK, Oh MJ, Wan J, Chen J, Smith KA, Fraidenburg DR, Choudhury MS, et al: Upregulated copper transporters in hypoxia-induced pulmonary hypertension. PLoS One 9: e90544, 2014.

65. Ranzer MJ, Chen L and DiPietro LA: Fibroblast function and wound breaking strength is impaired by acute ethanol intoxication. Alcohol Clin Exp Res 35: 83-90, 2011.

66. Shimoda LA and Semenza GL: HIF and the lung: Role of hypoxia-inducible factors in pulmonary development and disease. Am J Respir Crit Care Med 183: 152-156, 2011

67. Semenza GL: HIF-1: Mediator of physiological and pathophysiological responses to hypoxia. J Appl Physiol 88: 1474-1480, 2000

68. Adams JM, Difazio LT, Rolandelli RH, Luján JJ, Haskó G, Csóka B, Selmeczy Z and Németh ZH: HIF-1: A key mediator in hypoxia. Acta Physiol Hung 96: 19-28, 2009.
69. Higgins DF, Kimura K, Bernhardt WM, Shrimanker N, Akai Y, Hohenstein B, Saito Y, Johnson RS, Kretzler M, Cohen CD, et al: Hypoxia promotes fibrogenesis in vivo via HIF-1 stimulation of epithelial-to-mesenchymal transition. J Clin Invest 117: 3810-3820, 2007

70. Xia XD, Lee J, Khan S, Ye L, Li Y and Dong L: Suppression of Phosphatidylinositol 3-Kinase/Akt Signaling Attenuates Hypoxia-Induced Pulmonary Hypertension Through the Downregulation of Lysyl Oxidase. DNA Cell Biol 35: 599-606, 2016.

71. Nave AH, Mižíková I, Niess G, Steenbock H, Reichenberger F, Talavera ML, Veit F, Herold S, Mayer K, Vadász I, et al: Lysyl oxidases play a causal role in vascular remodeling in clinical and experimental pulmonary arterial hypertension. Arterioscler Thromb Vasc Biol 34: 1446-1458, 2014

72. Xiao Y, Nie X, Han P, Fu H and James Kang Y: Decreased copper concentrations but increased lysyl oxidase activity in ischemic hearts of rhesus monkeys. Metallomics 8: 973-980, 2016.

73. Mesarwi OA, Shin MK, Drager LF, Bevans-Fonti S, Jun JC, Putcha N, Torbenson MS, Pedrosa RP, Lorenzi-Filho G, Steele KE, et al: Lysyl Oxidase as a Serum Biomarker of Liver Fibrosis in Patients with Severe Obesity and Obstructive Sleep Apnea. Sleep (Basel) 38: 1583-1591, 2015.

74. Marturano JE, Xylas JF, Sridharan GV, Georgakoudi I and Kuo CK: Lysyl oxidase-mediated collagen crosslinks may be assessed as markers of functional properties of tendon tissue formation. Acta Biomater 10: 1370-1379, 2014.

75. Zhang Y, Wang B, Zhang WJ, Zhou G, Cao Y and Liu W: Enhanced proliferation capacity of porcine tenocytes in low $\mathrm{O}_{2}$ tension culture. Biotechnol Lett 32: 181-187, 2010.

76. Matafome P, Rodrigues T and Seica R: Glycation and Hypoxia: Two Key Factors for Adipose Tissue Dysfunction. Curr Med Chem 22: 2417-2437, 2015.

77. Reiser KM, Crouch EC, Chang K and Williamson JR: Lysyl oxidase-mediated crosslinking in granulation tissue collagen in two models of hyperglycemia. Biochim Biophys Acta 1097: 55-61, 1991.

78. Argyropoulos AJ, Robichaud P, Balimunkwe RM, Fisher GJ, Hammerberg C, Yan Y and Quan T: Alterations of Dermal Connective Tissue Collagen in Diabetes: Molecular Basis of Aged-Appearing Skin. PLoS One 11: e0153806, 2016.

79. Chronopoulos A, Tang A, Beglova E, Trackman PC and Roy S: High glucose increases lysyl oxidase expression and activity in retinal endothelial cells: Mechanism for compromised extracellular matrix barrier function. Diabetes 59: 3159-3166, 2010.

80. Yang X, Scott HA, Monickaraj F, Xu J, Ardekani S, Nitta CF, Cabrera A, McGuire PG, Mohideen U, Das A, et al: Basement membrane stiffening promotes retinal endothelial activation associated with diabetes. FASEB J 30: 601-611, 2016.

81. Rodríguez C, Raposo B, Martínez-González J, Casaní L and Badimon L: Low density lipoproteins downregulate lysyl oxidase in vascular endothelial cells and the arterial wall. Arterioscler Thromb Vasc Biol 22: 1409-1414, 2002.

82. Van Bergen $T$, Spangler R, Marshall D, Hollanders $K$, Van de Veire S, Vandewalle E, Moons L, Herman J, Smith V and Stalmans I: The Role of LOX and LOXL2 in the Pathogenesis of an Experimental Model of Choroidal Neovascularization. Invest Ophthalmol Vis Sci 56: 5280-5289, 2015.

83. Yang X, Scott HA, Ardekani S, Williams M, Talbot P and Ghosh K: Aberrant cell and basement membrane architecture contribute to sidestream smoke-induced choroidal endothelial dysfunction. Invest Ophthalmol Vis Sci 55: 3140-3147, 2014.

84. Kothapalli CR and Ramamurthi A: Lysyl oxidase enhances elastin synthesis and matrix formation by vascular smooth muscle cells. J Tissue Eng Regen Med 3: 655-661, 2009.

85. Cusella-De Angelis MG, Molinari S, Le Donne A, Coletta M, Vivarelli E, Bouche M, Molinaro M, Ferrari S and Cossu G: Differential response of embryonic and fetal myoblasts to TGF beta: A possible regulatory mechanism of skeletal muscle histogenesis. Development 120: 925-933, 1994.

86. Groenman FA, Rutter M, Wang J, Caniggia I, Tibboel D and Post M: Effect of chemical stabilizers of hypoxia-inducible factors on early lung development. Am J Physiol Lung Cell Mol Physiol 293: L557-L567, 2007.

87. Hong HH and Trackman PC: Cytokine regulation of gingival fibroblast lysyl oxidase, collagen, and elastin. J Periodontol 73 . $145-152,2002$

88. Huang G, Zhang Y, Kim B, Ge G, Annis DS, Mosher DF and Greenspan DS: Fibronectin binds and enhances the activity of bone morphogenetic protein 1. J Biol Chem 284: 25879-25888, 2009. 
89. Kishigami S and Mishina Y: BMP signaling and early embryonic patterning. Cytokine Growth Factor Rev 16: 265-278, 2005.

90. Pez F, Dayan F, Durivault J, Kaniewski B, Aimond G, Le Provost GS, Deux B, Clézardin P, SommerP, Pouysségur J, et al: The HIF-1-inducible lysyl oxidase activates HIF-1 via the Akt pathway in a positive regulation loop and synergizes with HIF-1 in promoting tumor cell growth. Cancer Res 71: 1647-1657, 2011.

91. Boak AM, Roy R, Berk J, Taylor L, Polgar P, Goldstein RH and Kagan HM: Regulation of lysyl oxidase expression in lung fibroblasts by transforming growth factor-beta 1 and prostaglandin E2. Am J Respir Cell Mol Biol 11: 751-755, 1994.

92. Xie J, Huang W, Jiang J, Zhang Y, Xu Y, Xu C, Yang L, Chen PC and Sung KL: Differential expressions of lysyl oxidase family in ACL and MCL fibroblasts after mechanical injury. Injury 44 : 893-900, 2013.

93. Leonard CM, Fuld HM, Frenz DA, Downie SA, Massagué J and Newman SA: Role of transforming growth factor-beta in chondrogenic pattern formation in the embryonic limb: Stimulation of mesenchymal condensation and fibronectin gene expression by exogenenous TGF-beta and evidence for endogenous TGF-beta-like activity. Dev Biol 145: 99-109, 1991.

94. Voloshenyuk TG, Landesman ES, Khoutorova E, Hart AD and Gardner JD: Induction of cardiac fibroblast lysyl oxidase by TGF- $\beta 1$ requires PI3K/Akt, Smad3, and MAPK signaling. Cytokine 55: 90-97, 2011.

95. Borel A, Eichenberger D, Farjanel J, Kessler E, Gleyzal C, Hulmes DJ, Sommer P and Font B: Lysyl oxidase-like protein from bovine aorta. Isolation and maturation to an active form by bone morphogenetic protein-1. J Biol Chem 276: 48944-48949, 2001.
96. Uzel MI, Scott IC, Babakhanlou-Chase H, Palamakumbura AH, Pappano WN, Hong HH, Greenspan DS and Trackman PC: Multiple bone morphogenetic protein 1-related mammalian metalloproteinases process pro-lysyl oxidase at the correct physiological site and control lysyl oxidase activation in mouse embryo fibroblast cultures. J Biol Chem 276: 22537-22543, 2001.

97. Fogelgren B, Polgár N, Szauter KM, Ujfaludi Z, Laczkó R, Fong KS and Csiszar K: Cellular fibronectin binds to lysyl oxidase with high affinity and is critical for its proteolytic activation. J Biol Chem 280: 24690-24697, 2005.

98. Liu X, Zhao Y, Pawlyk B, Damaser M and Li T: Failure of elastic fiber homeostasis leads to pelvic floor disorders. Am J Pathol 168: 519-528, 2006.

99. Sanada H, Shikata J, Hamamoto H, Ueba Y, Yamamuro T and Takeda T: Changes in collagen cross-linking and lysyl oxidase by estrogen. Biochim Biophys Acta 541: 408-413, 1978 .

100. Harlow CR, Rae M, Davidson L, Trackman PC and Hillier SG: Lysyl oxidase gene expression and enzyme activity in the rat ovary: Regulation by follicle-stimulating hormone, androgen, and transforming growth factor-beta superfamily members in vitro. Endocrinology 144: 154-162, 2003.

101. Slee RB, Hillier SG, Largue P, Harlow CR, Miele G and Clinton M: Differentiation-dependent expression of connective tissue growth factor and lysyl oxidase messenger ribonucleic acids in rat granulosa cells. Endocrinology 142: 1082-1089, 2001.

This work is licensed under a Creative Commons Attribution-NonCommercial-NoDerivatives 4.0 International (CC BY-NC-ND 4.0) License. 